[5] P. Y. Li and R. Horowitz, "Passive velocity field control of mechanical manipulators," IEEE Trans. Robot. Autom., vol. 15, no. 4, pp. 751-763, Aug. 1999.

[6] P. Y. Li and R. Horowitz, "Passive velocity field control (PVFC): Part I-Geometry and robustness," IEEE Trans. Autom. Control, vol. 46, no. 9, pp. 1346-1359, Sep. 2001.

[7] P. Y. Li and R. Horowitz, "Passive velocity field control (PVFC): Part II-Application to contour following," IEEE Trans. Autom. Control, vol. 46, no. 9, pp. 1360-1371, Sep. 2001.

[8] I. Cervantes, R. Kelly, J. Alvarez-Ramirez, and J. Moreno, "A robust velocity field control," IEEE Trans. Control Syst. Technol., vol. 10, no. 6, pp. 888-894, Nov. 2002.

[9] A. P. Aguiar and J. P. Hespanha, "Trajectory-tracking and path-following of underactuated autonomous vehicles with parametric modeling uncertainty," IEEE Trans. Autom. Control, vol. 52, no. 8, pp. 1362-1379, Aug. 2007.

[10] M. Y. Cheng and C. C. Lee, "Motion controller design for contour following tasks based on real-time contour error estimation," IEEE Trans. Ind. Electron., vol. 54, no. 3, pp. 1686-1695, Jun. 2007.

[11] R. K. Nagle and E. B. Saff, Fundamentals of Differential Equations and Boundary Value Problems. Reading, MA: Addison-Wesley, 1993.

[12] G. B. Gustafson and C. H. Wilcox, Analytical and Computational Methods of Advanced Engineering Mathematics. New York: Springer, 1998.

[13] J. F. Epperson, An Introduction to Numerical Methods and Analysis. New York: Wiley, 2002.

[14] P. V. O'Neil, Advanced Engineering Mathematics, 5th ed. Mason, OH: Thomson Learning, 2003.

[15] C. H. Edwards and D. E. Penney, Differential Equations \& Linear Algebra. Englewood Cliffs, NJ: Pearson Prentice Hall, 2005.

[16] L. Piegl, "On NURBS: A survey," IEEE Comput. Graph. Appl., vol. 11, no. 1, pp. 55-71, Jan. 1991.

[17] M. Y. Cheng, M. C. Tsai, and J. C. Kuo, "Real-time NURBS command generators for CNC servo controllers," Int. J. Mach. Tools Manufact., vol. 42, no. 7, pp. 801-813, May 2002.

[18] J. J. E. Slotine and W. Li, Applied Nonlinear Control. Englewood Cliffs, NJ: Prentice Hall, 1991.

[19] C. I. Byrnes and A. Isidori, "Limits, zero dynamics, and internal models in the problem of nonlinear output regulation," IEEE Trans. Autom. Control, vol. 48, no. 10, pp. 1712-1723, Oct. 2003.

[20] R. G. Bartle, The Elements of Real Analysis. New York: Wiley, 1976.

[21] V. F. Dem'yanov and V. N. Malozemov, Introduction to Minimax. New York: Wiley, 1974.

\section{Parameter-Dependent Lyapunov Functions for Linear Systems With Constant Uncertainties}

Peter Seiler, Ufuk Topcu, Andy Packard, and Gary Balas

\begin{abstract}
Robust stability of linear time-invariant systems with respect to structured uncertainties is considered. The small gain condition is sufficient to prove robust stability and scalings are typically used to reduce the conservatism of this condition. It is known that if the small gain condition is satisfied with constant scalings then there is a single quadratic Lyapunov function which proves robust stability with respect to all allowable time-varying perturbations. In this technical note we show that if the small gain condition is satisfied with frequency-varying scalings then an explicit parameter dependent Lyapunov function can be constructed to prove robust stability with respect to constant uncertainties. This Lyapunov function has a rational quadratic dependence on the uncertainties.
\end{abstract}

Index Terms-Parameter-dependent Lyapunov function (PDLF).

\section{INTRODUCTION}

Modeling uncertainties can be represented as parametric and/or dynamic perturbations to a nominal model. The entire collection of modeling uncertainties in a system can be collected into a single structured uncertainty (see, for example, [1], [2]). The structured singular value $(\mu)$ [2]-[4] provides a necessary and sufficient condition for robust stability with respect to structured linear-time invariant perturbations. However it is known that computing $\mu$ is NP Hard [5], [6]. Thus there has been extensive research into computational algorithms which are fast and provide good lower/upper bounds for most problems of engineering interest.

The small gain condition [7], [8] provides an easily computable sufficient condition for robust stability but is, in general, not necessary. Thus scalings are typically introduced to reduce the conservatism. For example, if there exists constant or frequency-dependent D-scales from an allowable set such that the small gain condition holds, then the system is robustly stable. These tests have their roots in the multiplier approaches used for passivity analysis [7]. In [2], these two conditions are referred to as the frequency domain constant $\mathrm{D}$ test and the frequency domain upper bound. In this technical note we will refer to these as the constant $\mathrm{D}$ and varying $\mathrm{D}$ tests.

The constant D test is necessary and sufficient for robust stability with respect to arbitrarily fast linear time-varying perturbations [9]. This condition is connected to the notion of quadratic stability, i.e., the existence of a single quadratic Lyapunov function which proves stability of all possible trajectories of the uncertain system for both fixed and time-varying perturbations [10]-[15]. In particular, if the small gain condition holds with constant D-scales then the uncertain system is quadratically stable.

Manuscript received March 05, 2009; revised June 17, 2009. First published September 22, 2009; current version published October 07, 2009. This work was supported in part under a NASA Langley NRA NNH077ZEA001N entitled "Analytical Validation Tools for Safety Critical Systems" and by the Air Force Office of Scientific Research, USAF, under Grant FA9550-05-1-0266. Recommended by Associate Editor H. Ito.

P. Seiler and G. Balas are with the University of Minnesota, Minneapolis, MN 55455 USA (e-mail: seiler@aem.umn.edu; balas@aem.umn.edu).

U. Topcu is with the California Institute of Technology, Pasadena, CA 91125 USA (e-mail: utopcu@cds.caltech.edu).

A. Packard is with the University of California at Berkeley, Berkeley, CA 94720 USA (e-mail: pack@me.berkeley.edu).

Color versions of one or more of the figures in this technical note are available online at http://ieeexplore.iee.org.

Digital Object Identifier 10.1109/TAC.2009.2029294 
The varying $\mathrm{D}$ test has a different interpretation. The use of frequency-varying D-scales renders the small gain condition necessary and sufficient for robust stability with respect to arbitrarily slowlyvarying linear perturbations [16]. This is the condition that is typically used when computing $\mu$ versus frequency. Clearly this test is also sufficient for robust stability with respect to all constant perturbations from the allowable set. Thus for each fixed perturbation there exists a Lyapunov function proving stability of the system. In contrast to the constant $\mathrm{D}$ test, this Lyapunov function may be a function of the perturbation. This technical note provides an explicit expression for a parameter-dependent Lyapunov function (PDLF) which can be derived from the varying D test. This Lyapunov function has a rational quadratic dependence on the uncertainties.

There is a significant amount of related research on PDLFs. The classical Popov criterion, when applied to a linear uncertainty, can be interpreted as using a PDLF to prove robust stability [14], [17]. This Lyapunov function is quadratic in the state and has an affine dependence on the uncertainty. There are many approaches to develop robust stability conditions using more general PDLFs. Lyapunov functions having an affine [14], [17]-[25], multi-affine [26], bi-quadratic [27], generic polynomial [28]-[31], and linear fractional dependence [32], [33] on the uncertainty have been considered. Hermite matrices [34] and power forms [35] have also been considered. The Kalman-Yakubovich-Popov (KYP) lemma [36] connects the PDLF in the Popov Criterion to a frequency domain condition but there are few additional connections for these more general PDLF conditions. One connection is made in [20], [21]. In particular, [20], [21] consider linear systems with affine dependence on real parameter uncertainties. They derive a sufficient condition for robust stability using a PDLF having affine dependence on the uncertainties. They demonstrate that this sufficient condition is equivalent to the standard real $\mu$ upper bound [37] but restricted to have constant $D$ scales and $G$ scales having a specific affine dependence on frequency. Thus the condition in [20], [21] is more conservative than the real $\mu$ upper bound with generic frequency-varying $D-G$ scales which is known to be equal to $\mu$ for certain block structures [38].

The work on quadratic separators provides another relevant connection to PDLFs [39]-[42]. The authors derive necessary and sufficient robust stability conditions based on finding a Hermitian matrix-valued function, termed a quadratic separator, which topologically separates the graph of the nominal system from the inverse graph of each uncertainty in the allowable set. One version of this condition can be interpreted as simultaneously searching for a PDLF and a parameter-dependent quadratic separator which satisfy a linear matrix inequality (see Theorem 3 of [40]). These necessary and sufficient conditions are computationally difficult to solve (they are equivalent to computing $\mu$ ) and hence various sufficient conditions are derived. One of these sufficient conditions, termed the vertex-separator condition, can be used to construct a PDLF with polytopic dependence on the uncertainties. Another sufficient condition is obtained for linear parameter varying systems by applying a constant quadratic separator. This sufficient condition is shown to be equivalent to the existence of a PDLF which has a linear fractional dependence on the uncertainty (see Theorem 4 of [42]). This particular PDLF will be discussed further in Section IV.

\section{NOTATION}

$\mathbb{C}^{n \times m}$ and $\mathbb{R}^{n \times m}$ are complex and real $n \times m$ matrices, respectively. For $M \in \mathbb{C}^{n \times m}, M^{*}$ is the complex conjugate transpose of $M$. The maximum singular value of $M$ is denoted by $\bar{\sigma}(M)$. For $M \in \mathbb{C}^{n \times n}$, the spectral radius of $M$ is denoted by $\rho(M)$. If $M=M^{*}$ then all the eigenvalues of $M$ are real and $\lambda_{\max }(M)$ denotes the largest eigenvalue. If $M=M^{*}$ then $M>0$ and $M<0$ denote the matrix is positive and negative definite, respectively. Given $A \in \mathbb{C}^{n \times m}$ and $B \in \mathbb{C}^{r \times s}, \operatorname{diag}(A, B) \in \mathbb{C}^{(n+r) \times(m+s)}$ denotes the block diagonal concatenation of the two matrices. $A \otimes B \in \mathbb{C}^{r n \times s m}$ denotes the Kronecker product. Let $n$ and $m$ be positive integers and partition $M \in$ $\mathbb{C}^{(n+m) \times(n+m)}$ as $M=\left[\begin{array}{ll}A & B \\ C & D\end{array}\right]$ where $A \in \mathbb{C}^{n \times n}, B \in \mathbb{C}^{n \times m}$, $C \in \mathbb{C}^{m \times n}$, and $D \in \mathbb{C}^{m \times m}$. Let $\Delta \in \mathbb{C}^{m \times m}$ be a matrix such that $I-D \Delta$ is invertible. In this case we define the linear fractional transformation $F_{l}(M, \Delta):=A+B \Delta(I-D \Delta)^{-1} C$. The subscript $l$ refers to the closure of the lower block of $M$ with the matrix $\Delta$ and we can use this transformation to define an uncertain, autonomous discrete-time system: $x_{k+1}=F_{l}(M, \Delta) x_{k}$. Similarly, for $\Omega \in \mathbb{C}^{n \times n}$ such that $I-A \Omega$ is invertible, we define $F_{u}(M, \Omega):=D+C \Omega(I-A \Omega)^{-1} B$. The subscript $u$ refers to the upper block of $M$ being closed with the matrix $\Omega$. This transformation can be used to define a transfer function matrix, e.g., $G(z):=D+C\left(z I_{n}-A\right)^{-1} B=F_{u}\left(M, \frac{1}{z} I_{n}\right)$. We define $\|G\|_{\infty}:=\max _{0 \leq \theta \leq 2 \pi} \bar{\sigma}\left(G\left(e^{j \theta}\right)\right)$.

\section{PRELIMINARY RESUlTS}

This section presents lemmas which are used in Section IV to construct a PDLF from the varying D test. The first lemma is the Schur complement lemma. The next lemma relates a block $2 \times 2$ Lyapunov inequality to Lyapunov inequalities for the diagonal blocks. The last lemma relates an algebraic Riccati inequality to a robust stability condition.

Lemma 1 (Schur Complements [14], [43]): Let $P:=\left[\begin{array}{ll}P_{11} & P_{12} \\ P_{12}^{*} & P_{22}\end{array}\right] \in \mathbb{C}^{(n+m) \times(n+m)}$ and $P=P^{*}$. The following conditions are equivalent:

A) $P>0$;

B) $P_{11}>0$ and $P_{22}-P_{12}^{*} P_{11}^{-1} P_{12}>0$;

C) $P_{22}>0$ and $P_{11}-P_{12} P_{22}^{-1} P_{12}^{*}>0$.

Lemma 2 (Block Lyapunov Inequality): Let $A:=\left[\begin{array}{cc}A_{11} & A_{12} \\ 0 & A_{22}\end{array}\right] \in$ $\mathbb{C}^{(n+m) \times(n+m)}$ and $P:=\left[\begin{array}{ll}P_{11} & P_{12} \\ P_{12}^{*} & P_{22}\end{array}\right] \in \mathbb{C}^{(n+m) \times(n+m)}$ be partitioned conformably. If $P=P^{*}>0$ and $A^{*} P A-P<0$ then:

A) $\hat{P}:=P_{11}$ satisfies $\hat{P}>0$ and $A_{11}^{*} \hat{P} A_{11}-\hat{P}<0$;

B) $\hat{Q}:=P_{22}-P_{12}^{*} P_{11}^{-1} P_{12}$ satisfies $\hat{Q}>0$ and $A_{22}^{*} \hat{Q} A_{22}-\hat{Q}<0$. Proof:

A) Any diagonal block of a positive (negative) definite matrix must itself be positive (negative) definite. Thus $P>0$ implies $\hat{P}>0$. Also, the $(1,1)$ block of $A^{*} P A-P$ is $A_{11}^{*} \hat{P} A_{11}-\hat{P}$ and hence this quantity is negative definite.

B) By the Schur complement lemma, $P>0$ implies $\hat{Q}>0$. Also by the Schur complement lemma, $A^{*} P A-P<0$ implies $A P^{-1} A^{*}-P^{-1}<0$. The $(2,2)$ block of $P^{-1}$ is $\hat{Q}^{-1}$ (see Equation A.1.7 of [43]). Thus the (2,2) block of $A P^{-1} A^{*}-P^{-1}<0$ implies $A_{22} \hat{Q}^{-1} A_{22}^{*}-\hat{Q}^{-1}<0$. One more application of the Schur complement lemma brings the desired result, $A_{22}^{*} \hat{Q} A_{22}-$

$\hat{Q}<0$.
Lemma 3 (Robust Stability Condition): Let $M:=\left[\begin{array}{cc}A & B \\ C & D\end{array}\right] \in$ $\mathbb{C}^{(n+m) \times(n+m)}$. If there exists $P=P^{*} \in \mathbb{C}^{n \times n}$ such that $P>0$ and $A^{*} P A-P+Z^{*} Y^{-1} Z+C^{*} C<0$

where $Y:=I-D^{*} D-B^{*} P B$ and $Z:=B^{*} P A+D^{*} C$ then $P$ satisfies

$$
\max _{\Delta \in \mathbb{C}^{m \times m, \bar{\sigma}(\Delta) \leq 1}} \lambda_{\max }\left(F_{l}(M, \Delta)^{*} P F_{l}(M, \Delta)-P\right)<0 .
$$

Proof: The S-procedure can be used to prove this lemma. For example, Chapter 5 of [14] applies the S-procedure to derive a robust stability condition for continuous-time LTI systems with norm-bounded time-varying uncertainties. For completeness, we provide a proof for discrete-time systems which is based on completion of a square. 
The algebraic Riccati inequality (1) can be used to show $\bar{\sigma}(D)<1$. Thus $I-D \Delta$ is invertible and $F_{l}(M, \Delta)$ is well-defined for all $\Delta$ with $\bar{\sigma}(\Delta) \leq 1$. To simplify notation, define $W:=\Delta(I-D \Delta)^{-1}$ so that $F_{l}(M, \Delta)=A+B W C$. Completing a square and using $\bar{\sigma}(\Delta) \leq 1$ yields

$$
\begin{aligned}
F_{l}( & M, \Delta)^{*} P F_{l}(M, \Delta)-P \\
= & A^{*} P A-P+Z^{*} Y^{-1} Z-(Y W C-Z)^{*} Y^{-1}(Y W C-Z) \\
& +C^{*}\left[W^{*} W-W^{*} D^{*} D W-W^{*} D^{*}-D W\right] C \\
= & A^{*} P A-P+Z^{*} Y^{-1} Z-(Y W C-Z)^{*} Y^{-1}(Y W C-Z) \\
& +\left[C^{*} C+C^{*}(I-D \Delta)^{-*}\left(\Delta^{*} \Delta-I\right)(I-D \Delta)^{-1} C\right] \\
\leq & A^{*} P A-P+Z^{*} Y^{-1} Z+C^{*} C .
\end{aligned}
$$

The desired result follows by applying (1).

\section{MAIN RESULT}

In this section we consider the robust stability of a discrete time system with respect to structured uncertainties. We consider block structures $\Delta \subset \mathbb{C}^{m \times m}$ consisting of $s$ repeated complex scalar blocks and $f$ square full complex blocks. The restriction to square full blocks is for notational simplicity. Given positive integers $m_{1}, m_{2}, \ldots, m_{s+f}$ satisfying $\sum_{i=1}^{s+f} m_{i}=m$, we can define the following sets of block structured $m \times m$ matrices:

$$
\begin{aligned}
\Delta:= & \left\{\Delta=\operatorname{diag}\left(\delta_{1} I_{m_{1}}, \ldots, \delta_{s} I_{m_{s}}, \Delta_{1}, \ldots, \Delta_{f}\right):\right. \\
& \left.\delta_{i} \in \mathbb{C}, \Delta_{i} \in \mathbb{C}^{m_{s+i} \times m_{s+i}}\right\} \\
B_{\Delta}:= & \{\Delta \in \Delta: \bar{\sigma}(\Delta) \leq 1\} .
\end{aligned}
$$

Associated with these block structures we can define sets of constant and varying $\mathrm{D}$-scales

$$
\begin{aligned}
\mathbb{D}_{c}:= & \left\{D_{c} \in \mathbb{C}^{m \times m}: D_{c} \Delta=\Delta D_{c} \forall \Delta \in \Delta, \operatorname{det}\left(D_{c}\right) \neq 0\right\} \\
\mathbb{D}_{v}:= & \left\{D_{v}(z):=D_{d}+C_{d}\left(z I-A_{d}\right)^{-1} B_{d}: \operatorname{det}\left(D_{d}\right) \neq 0,\right. \\
& \left.\quad\left[\begin{array}{ll}
A_{d} & B_{d} \\
C_{d} & D_{d}
\end{array}\right] \in \mathbb{C}^{(k+m) \times(k+m)}, D_{v} \Delta=\Delta D_{v} \forall \Delta \in \Delta\right\} .
\end{aligned}
$$

By definition, any scaling $D_{s}$ from either $\mathbb{D}_{c}$ or $\mathbb{D}_{v}$ satisfies $\Delta=$ $D_{s}^{-1} \Delta D_{s} \forall \Delta \in \Delta$. Thus we can insert $D_{s}$ at the input to $\Delta$ and $D_{s}^{-1}$ at the output of $\Delta$. If $\left\|D_{s} F_{u}\left(M, \frac{1}{z} I\right) D_{s}^{-1}\right\|_{\infty}<1$ then $x_{k+1}=$ $F_{l}\left(M, D_{s}^{-1} \Delta D_{s}\right) x_{k}$ is robustly stable with respect to $B_{\Delta}$. By the equivalence of the scaled and unscaled systems, we can then conclude that $x_{k+1}=F_{l}(M, \Delta) x_{k}$ is robustly stable with respect to $B_{\Delta}$. This is an indirect proof of robust stability. The two theorems in this section directly prove robust stability by constructing Lyapunov functions for $x_{k+1}=F_{l}(M, \Delta) x_{k}$. The theorems are stated in a form which highlights this construction.

Theorem 1 (Constant D Test): Let $G(z):=F_{u}\left(M, \frac{1}{z} I_{n}\right)$ where $M:=\left[\begin{array}{ll}A & B \\ C & D\end{array}\right] \in \mathbb{C}^{(n+m) \times(n+m)}$ and $\rho(A)<1$. If there exists $D_{c} \in \mathbb{D}_{c}$ such that $\left\|D_{c} G D_{c}^{-1}\right\|_{\infty}<1$ then:

A) There exists $P=P^{*} \in C^{n \times n}$ such that $P>0$ and

$$
\begin{aligned}
\tilde{A}^{*} P \tilde{A}-P & +\tilde{C}^{*} \tilde{C}+\left(\tilde{B}^{*} P \tilde{A}+\tilde{D}^{*} \tilde{C}\right)^{*} \\
& \times\left(I-\tilde{D}^{*} \tilde{D}-\tilde{B}^{*} P \tilde{B}\right)^{-1}\left(\tilde{B}^{*} P \tilde{A}+\tilde{D}^{*} \tilde{C}\right)<0
\end{aligned}
$$

where $\tilde{A}:=A, \tilde{B}:=B D_{c}^{-1}, \tilde{C}:=D_{c} C$, and $\tilde{D}:=D_{c} D D_{c}^{-1}$.

B) The solution $P>0$ to (5) satisfies

$$
\max _{\Delta \in B_{\Delta}} \lambda_{\max }\left(F_{l}(M, \Delta)^{*} P F_{l}(M, \Delta)-P\right)<0 .
$$

C) Let $\left\{\Delta_{k}\right\}_{k=0}^{\infty} \subset B_{\Delta}$ be given. Then $x=0$ is a globally exponentially stable equilibrium point of $x_{k+1}=F_{l}\left(M, \Delta_{k}\right) x_{k}$. Proof:

A) The $\tilde{A}, \tilde{B}, \tilde{C}$, and $\tilde{D}$ given in the theorem are the state matrices for the system $D_{c} G D_{c}^{-1}$. The existence of $P>0$ satisfying (5) follows from $\left\|D_{c} G D_{c}^{-1}\right\|_{\infty}<1$ and the discrete-time Bounded Real Lemma [2].

B) Define $\tilde{M}=\left[\begin{array}{ll}A & \tilde{B} \\ \tilde{C} & \tilde{D}\end{array}\right]$. Since $P>0$ satisfies (5), we can apply Lemma 3 to conclude

$$
\max _{\Delta \in \mathbb{C}^{m \times m}, \bar{\sigma}(\Delta) \leq 1} \lambda_{\max }\left(F_{l}(\tilde{M}, \Delta)^{*} P F_{l}(\tilde{M}, \Delta)-P\right)<0 .
$$

Restricting to $\Delta \in B_{\Delta}$, we can use $D_{c} \Delta=\Delta D_{c}$ to show $F_{l}(\tilde{M}, \Delta)=F_{l}(M, \Delta)$.

C) Define the Lyapunov function $V(x)=x^{T} P x$. B) implies there exists a $\beta<1$ such that for any $\Delta_{k} \in B_{\Delta}, V\left(x_{k+1}\right)<\beta V\left(x_{k}\right)$ which guarantees the robust stability with respect to time-varying perturbations. Formally, C) follows from B) and discrete-time Lyapunov theory (Section 5.9 of [44]).

As noted in the introduction, an uncertain system is quadratically stable if there exists a single quadratic Lyapunov function which proves stability of all possible trajectories of the uncertain system. Theorem 1 demonstrates that satisfying the small-gain theorem with constant D-scales implies quadratic stability. The next theorem demonstrates that using frequency varying D-scales implies the existence of a PDLF. One difficulty is that the state matrices of $D_{v}(z) \in \mathbb{D}_{v}$ do not commute with the $\Delta \in \boldsymbol{\Delta}$. However, we can derive how $\Delta$ is altered as it "moves through" the state matrices of $D_{v}(z)$.

Let $D_{v}(z):=D_{d}+C_{d}\left(z I-A_{d}\right)^{-1} B_{d}$. Since $D_{v}(z)$ commutes with all $\Delta \in \Delta$, it must be block-diagonal

$D_{v}(z)=\operatorname{diag}\left(D_{v, 1}(z), \ldots, D_{v, s}(z)\right.$,

$$
\left.d_{v, s+1}(z) I_{m_{s+1}}, \ldots, d_{v, s+f}(z) I_{m_{s+f}}\right) \text {. }
$$

A natural state space realization for $D_{v}(z)$ is given by

$$
\begin{gathered}
A_{d}:=\operatorname{diag}\left(A_{d, 1}, \ldots, A_{d, s},\left(I_{m_{s+1}} \otimes A_{d, s+1}\right),\right. \\
\left.\ldots,\left(I_{m_{s+f}} \otimes A_{d, s+f}\right)\right) \\
B_{d}:=\operatorname{diag}\left(B_{d, 1}, \ldots, B_{d, s},\left(I_{m_{s+1}} \otimes B_{d, s+1}\right),\right. \\
\left.\ldots,\left(I_{m_{s+f}} \otimes B_{d, s+f}\right)\right) \\
C_{d}:=\operatorname{diag}\left(C_{d, 1}, \ldots, C_{d, s},\left(I_{m_{s+1}} \otimes C_{d, s+1}\right),\right. \\
\left.\ldots,\left(I_{m_{s+f}} \otimes C_{d, s+f}\right)\right) \\
D_{d}:=\operatorname{diag}\left(D_{d, 1}, \ldots, D_{d, s},\left(I_{m_{s+1}} \otimes D_{d, s+1}\right),\right. \\
\left.\ldots,\left(I_{m_{s+f}} \otimes D_{d, s+f}\right)\right)
\end{gathered}
$$

where $\left[\begin{array}{ll}A_{d, i} & B_{d, i} \\ C_{d, i} & D_{d, i}\end{array}\right] \in \mathbb{C}^{\left(k_{i}+m_{i}\right) \times\left(k_{i}+m_{i}\right)}$ are the state space matrices of $D_{v, i}(z)(i=1, \ldots, s)$ and $\left[\begin{array}{ll}A_{d, i} & B_{d, i} \\ C_{d, i} & D_{d, i}\end{array}\right] \in$ $\mathbb{C}^{\left(k_{i}+1\right) \times\left(k_{i}+1\right)}$ are the state space matrices of $d_{v, i}(z)(i=$ $s+1, \ldots, s+f)$. Next, define $X(\Delta): \Delta \rightarrow \mathbb{C}^{k \times k}$ by

$X(\Delta)=\operatorname{diag}\left(\delta_{1} I_{k_{1}}, \ldots, \delta_{s} I_{k_{s}}, \Delta_{s+1} \otimes I_{k_{s+1}}\right.$,

$$
\left.\ldots, \Delta_{s+f} \otimes I_{k_{s+f}}\right) .
$$

The dimension of the $i$ th block of $X(\Delta)$ depends on the state dimension, $k_{i}$, of the transfer function in $i$ th block of the $D_{v}(z)$. For any $\Delta \in \boldsymbol{\Delta}$, the state space realization of $D_{v}(z)$ given in (7)-(10) satisfies the commutation relations: $A_{d} X(\Delta)=X(\Delta) A_{d}, B_{d} \Delta=X(\Delta) B_{d}$, 
$C_{d} X(\Delta)=\Delta C_{d}$, and $D_{d} \Delta=\Delta D_{d}$. Thus $\Delta$ commutes with $D_{d}$ but is altered when passing through $B_{d}$ and $C_{d}$. These relations will be used in the proof of the following theorem.

Theorem 2 (Varying $D$ Test): Let $M:=\left[\begin{array}{cc}A & B \\ C & D\end{array}\right] \in$ $\mathbb{C}^{(n+m) \times(n+m)}$ with $\rho(A)<1$ and define $G(z):=F_{u}\left(M, \frac{1}{z} I_{n}\right)$. If there exists $D_{v}(z) \in \mathbb{D}_{v}$ such that $\left\|D_{v} G D_{v}^{-1}\right\|_{\infty}<1$ then:

A) There exists $P=P^{*} \in C^{(n+2 k) \times(n+2 k)}$ such that $P>0$ and

$$
\begin{aligned}
\tilde{A}^{*} P \tilde{A}-P+ & \left(\tilde{B}^{*} P \tilde{A}+\tilde{D}^{*} \tilde{C}\right)^{*}\left(I-\tilde{D}^{*} \tilde{D}-\tilde{B}^{*} P \tilde{B}\right)^{-1} \\
& \left(\tilde{B}^{*} P \tilde{A}+\tilde{D}^{*} \tilde{C}\right)+\tilde{C}^{*} \tilde{C}<0
\end{aligned}
$$

where $\left[\begin{array}{ll}A_{d} & B_{d} \\ C_{d} & D_{d}\end{array}\right] \in \mathbb{C}^{(k+m) \times(k+m)}$ are the state matrices of
$D_{v}(z)$ and

$$
\begin{aligned}
& \tilde{A}:=\left[\begin{array}{ccc}
A_{d} & B_{d} C & -B_{d} D D_{d}^{-1} C_{d} \\
0 & A & -B D_{d}^{-1} C_{d} \\
0 & 0 & A_{d}-B_{d} D_{d}^{-1} C_{d}
\end{array}\right], \\
& \tilde{B}:=\left[\begin{array}{c}
B_{d} D D_{d}^{-1} \\
B D_{d}^{-1} \\
B_{d} D_{d}^{-1}
\end{array}\right] \\
& \tilde{C}:=\left[\begin{array}{lll}
C_{d} & D_{d} C & -D_{d} D D_{d}^{-1} C_{d}
\end{array}\right], \\
& \tilde{D}:=D_{d} D D_{d}^{-1} \text {. }
\end{aligned}
$$

B) Given the solution $P>0$ to (11), define

$$
\begin{aligned}
\hat{P}(\Delta):= & P_{22}+\left(P_{21}+P_{23} X(\Delta)\right)\left(P_{11}+P_{13} X(\Delta)\right. \\
& \left.+X(\Delta)^{*} P_{13}^{*}+X(\Delta)^{*} X_{33} X(\Delta)\right)^{-1} \\
& \times\left(P_{21}+P_{23} X(\Delta)\right)^{*}
\end{aligned}
$$

where the $P_{i j}$ are a block $3 \times 3$ partition of $P$ conformable with the $3 \times 3$ blocks of $\tilde{A}$. Then $\hat{P}(\Delta)>0 \forall \Delta \in B_{\Delta}$ and

$\max _{\Delta \in B_{\Delta}} \lambda_{\max }\left(F_{l}(M, \Delta)^{*} \hat{P}(\Delta) F_{l}(M, \Delta)-\hat{P}(\Delta)\right)<0$.

C) Let $\Delta \in B_{\Delta}$ be given. Then $x=0$ is a globally exponentially stable equilibrium point of $x_{k+1}=F_{l}(M, \Delta) x_{k}$.

Proof:

A) The $\tilde{A}, \tilde{B}, \tilde{C}$, and $\tilde{D}$ given in the theorem statement are the state matrices for the system $D_{v} G D_{v}^{-1}$. The existence of $P>0$ satisfying (11) follows from $\left\|D_{v} G D_{v}^{-1}\right\|_{\infty}<1$ and the discrete-time Bounded Real Lemma [2].

B) Define $\tilde{M}=\left[\begin{array}{ll}\tilde{A} & \tilde{B} \\ \tilde{C} & \tilde{D}\end{array}\right]$. Since $P>0$ satisfies (11), we can apply Lemma 3 to conclude

$$
\max _{\Delta \in \mathbb{C}^{m \times m}, \bar{\sigma}(\Delta) \leq 1} \lambda_{\max } \times\left(F_{l}(\tilde{M}, \Delta)^{*} P F_{l}(\tilde{M}, \Delta)-P\right)<0 .
$$

Define the coordinate transformation

$$
T:=\left[\begin{array}{ccc}
I & 0 & 0 \\
0 & I & 0 \\
X(\Delta) & 0 & I
\end{array}\right]
$$

Define $R:=T^{-1} F_{l}(\tilde{M}, \Delta) T$ and $S:=T^{*} P T$. Multiplying $F_{l}(\tilde{M}, \Delta)^{*} P F_{l}(\tilde{M}, \Delta)-P$ on the left/right by $T^{*} / T$ and inserting $T T^{-1}$ yields $R^{*} S R-S$. This is a congruence transformation and hence $R^{*} S R-S$ remains strictly negative definite for all $\Delta \in B_{\Delta}$. Performing block multiplications and applying the commutations relations satisfied by $X(\Delta)$ yields

$$
\begin{aligned}
R & =\left[\begin{array}{ccc}
(\cdot) & (\cdot) & (\cdot) \\
0 & F_{l}(M, \Delta) & (\cdot) \\
0 & 0 & (\cdot)
\end{array}\right] \\
S & =\left[\begin{array}{ccc}
S_{11} & \left(P_{21}+P_{23} X(\Delta)\right)^{*} & (\cdot) \\
P_{21}+P_{23} X(\Delta) & P_{22} & (\cdot) \\
(\cdot) & (\cdot) & (\cdot)
\end{array}\right]
\end{aligned}
$$

where $S_{11}:=P_{11}+P_{13} X(\Delta)+X(\Delta)^{*} P_{13}^{*}+X(\Delta)^{*} P_{33} X(\Delta)$ and blocks denoted by $(\cdot)$ do not affect the remaining argument in the proof. $S>0$ since it is related to $P>0$ by a nonsingular congruence transformation and hence $\hat{P}(\Delta)>0$ by the Schur complement lemma. The proof is concluded by first applying Lemma 2-A to $R^{*} S R-S<0$ to obtain inequalities in terms of the upper left $2 \times 2$ blocks of $R$ and $S$ and then applying Lemma 2-B.

C) For each $\Delta \in B_{\Delta}$ define the Lyapunov function $V(x, \Delta)=$ $x^{T} \hat{P}(\Delta) x$. C) follows from B) and discrete-time Lyapunov theory (Section 5.9 of [44]).

Comments:

- This technical note uses a discrete-time formulation but the results carry over to the continuous-time case. The lemmas in Section III must be restated in terms of the continuous-time Lyapunov inequality, Bounded-Real Lemma, and algebraic Riccati inequality (refer to Chapter 5 of [14]). The proofs and results in Section IV then require only minor modifications. The continuous-time PDLF in the varying D test has the same structure and dependence on the solution of the continuous-time algebraic Riccati inequality.

- The algebraic Riccati inequalities ((5) and (11)) can be converted to linear matrix inequalities by the Schur complement lemma. Thus we can use available software (e.g., LMILab [1] and Sedumi [45]) to solve for $P>0$. Both theorems then give an explicit construction for a Lyapunov function which proves robust stability. However, this can be computationally demanding in the case of the varying $\mathrm{D}$ test since the variable $P$ has dimension $n+2 k$ where $k$ is the state dimension of $D_{v}(z)$. Fitting the magnitude data, $\left|D_{v}\left(e^{\theta}\right)\right|$, from a frequency gridded mussv [1] calculation with a state-space model can lead to high state dimensions for $D_{v}(z)$. This is especially true for the full blocks of $D_{v}(z)$ associated with repeated scalar uncertainties.

- If the $i$ th block of $D_{v}(z)$ is constant then its state dimension is $k_{i}=0$. In this case $X(\Delta)$, and hence $P(\Delta)$, do not depend on the corresponding block of $\Delta$. Thus we can obtain Lyapunoy functions which are partially parameter-dependent by fixing some blocks of the D-scale to be constant and others to be frequency varying. The example in the following section will further demonstrate this point.

- PDLFs with polynomial dependence on the uncertainty are used in [28]-[31] for linear robust stability analysis and in [46]-[49] for nonlinear region of attraction analysis. While polynomial dependence is without loss of generality for linear robust stability [25], [50], it might require a high degree. It would be useful to see if algorithms can be developed based on the particular form of the PDLF given in (12).

Theorem 2 demonstrates that satisfying the small gain condition with frequency varying D-scales implies the existence of a PDLF with a rational-quadratic dependence on the uncertainties. The class of PDLFs of this form includes those which have an affine dependence on the uncertainties. This provides another explanation for why the affine PDLF condition given in [20], [21] is more conservative than using frequencyvarying $D-G$ scales. 
We can also compare the PDLF from the varying D test to that obtained from a special case of the quadratic separator condition for continuous-time, linear parameter varying systems [42]. These systems have the form $\dot{x}=A+B(I-\Delta(t) D)^{-1} \Delta(t) C$ with $\Delta(t)$ having a block diagonal structure of repeated real scalars. A sufficient condition for robust stability with respect to the time-varying real parameters is derived using a constant quadratic separator. This sufficient condition is no more conservative than using constant $D-G$ scales but it is, in general, more conservative than using a frequency varying quadratic separator. The sufficient condition with the constant quadratic separator is shown to be equivalent to the existence of a PDLF of the form

$$
\hat{P}(\Delta):=\left[\begin{array}{c}
I \\
(I-\Delta D)^{-1} \Delta C
\end{array}\right]^{T} P\left[\begin{array}{c}
I \\
(I-\Delta D)^{-1} \Delta C
\end{array}\right] .
$$

This PDLF proves robust stability with respect to time-varying parameter variations and hence it also proves robust stability with respect to constant parameter uncertainties. The class of PDLFs of this form is not directly comparable to PDLFs of the form given in (12); in general neither form is more general than the other. It is notable that if the nominal system has no direct feedthrough $(D=0)$ then the PDLF from the constant quadratic separator condition reduces to a quadratic dependence on the uncertainty. The form of the PDLF from the varying $\mathrm{D}$ test can, in principle, have a rational quadratic dependence on the uncertainty for any nominal system.

We can briefly summarize four related conditions for robustness with respect to constant uncertainties:

1) Constant D-Scales: The small-gain condition with constant $D$-scales is only a sufficient condition for robust stability. If this sufficient condition is satisfied then the system is quadratically stable and there exists a parameter independent Lyapunov function which proves robust stability.

2) Frequency Varying D-Scales: The small-gain condition with frequency varying $D$-scales is only a sufficient condition for robust stability. In this technical note we showed that if this sufficient condition is satisfied then one can explicitly construct a PDLF (12) which proves robust stability.

3) Constant Quadratic Separator: The constant quadratic separator condition is only a sufficient condition for robust stability. If this sufficient condition is satisfied then there is a PDLF (14) which proves robust stability [42]. In general, the form of this PDLF is neither more nor less general than the form derived from the varying $D$ test.

4) Frequency Varying Quadratic Separator: The frequency varying quadratic separator condition is necessary and sufficient for robust stability (see Theorem 1 in either [40] or [41]). It is not known how to explicitly construct a PDLF when this condition is satisfied. This would be interesting since it would provide a form for the PDLF which could be assumed without loss of generality when analyzing the robustness of linear systems with respect to constant uncertainties. In particular, it is well known that quadratic Lyapunov functions are sufficient to prove stability of linear systems. Thus if a linear system is stable then there is a Lyapunov function which is a quadratic function of the state which proves stability. One does not need to consider more complicated Lyapunov functions for linear systems. For stability analysis of uncertain linear systems this implies that we only need to consider Lyapunov functions which are quadratic in the state but with an arbitrary functional dependence on the uncertainty. It would be useful for algorithm development to know if there is a functional dependence on the uncertainties which can be assumed without loss of generality. Since the frequency-varying quadratic separator condition is necessary and sufficient for robust stability, it potentially provides a path to understanding this functional dependence. Specifically, if we can construct an explicit PDLF when this condition is satisfied then the form of this PDLF can be assumed without loss of generality when analyzing linear uncertain systems. One might then develop algorithms based on this functional form similar to the current development of algorithms centered around affine Lyapunov functions.

\section{EXAMPLE}

Consider the two-state system $x_{k+1}=F_{l}(M, \Delta) x_{k}$ from [12] where $\boldsymbol{\Delta}:=\left\{\Delta=\operatorname{diag}\left(\delta_{1}, \delta_{2}\right): \delta_{i} \in \mathbb{C}\right\}$ and

$$
M:=\left[\begin{array}{cccc}
a & 0 & 2 b a & 0 \\
0 & -a & 0 & -2 b a \\
0 & 1 & 0 & b \\
1 & 0 & b & 0
\end{array}\right] .
$$

Choose $a=0.9$ and $b=0.5$. This system is not quadratically stable but is robustly stable with respect to constant $\Delta \in \Delta$.

Consider the time-varying perturbations $\Delta_{k}=\operatorname{diag}(1,0)$ for $k$ even and $\Delta_{k}=\operatorname{diag}(0,-1)$ for $k$ odd. For $k$ odd, the two step evolution of the system is $x_{k+1}=\left[\begin{array}{cc}1.62 & -0.81 \\ -0.81 & 0.81\end{array}\right] x_{k}$. This has eigenvalues at 0.3094 and 2.1206 which demonstrates that the system is not stable for all time varying perturbations in $B_{\boldsymbol{\Delta}}$. Hence, the system is not quadratically stable. Define $G(z):=F_{u}\left(M, \frac{1}{z} I_{2}\right)$. We used LMILab [1] to minimize $\left\|D_{c} G D_{c}^{-1}\right\|_{\infty}$ over $D_{c} \in \mathbb{D}_{c}$. The optimal constant scaling is $D_{c}=I_{2}$ and, as expected, the minimal value of $\left\|D_{c} G D_{c}^{-1}\right\|_{\infty}=9.50$ which exceeds 1 .

Next, consider the scaling $D_{v}(z)=\operatorname{diag}\left(1, \frac{0.9975 z-0.9025}{z+0.9}\right)$. For this scaling $\left\|D_{v} G D_{v}^{-1}\right\|_{\infty}=0.526<1$ and by the varying $\mathrm{D}$ test we conclude the system is robustly stable with respect to constant $\Delta \in$ $B_{\Delta}$. We again used LMILab to compute $P>0$ which satisfies (11). The result is

$P=\left[\begin{array}{c|cc|c}1204.5 & -669.2 & 0 & 0 \\ \hline-669.2 & 371.9 & 0 & 0 \\ 0 & 0 & 789.8 & 712.6 \\ \hline 0 & 0 & 712.6 & 643.1\end{array}\right]$

The lines denote the $3 \times 3$ block partition used in the Lyapunov function construction of Theorem 2 . Using this construction, define the Lyapunov function $V(x, \Delta):=x^{T} \hat{P}(\Delta) x$ where $\hat{P}(\Delta)$ is given by

$$
\begin{aligned}
\hat{P}(\Delta)=\left[\begin{array}{cc}
371.9 & 0 \\
0 & 789.8
\end{array}\right] & \\
& +\frac{10^{5}}{643.1 \delta_{2}^{2}+1204.5}\left[\begin{array}{cc}
4.48 & -4.77 \delta_{2} \\
-4.77 \delta_{2} & 5.08 \delta_{2}^{2}
\end{array}\right] .
\end{aligned}
$$

This PDLF proves stability of $x_{k+1}=F_{l}(M, \Delta) x_{k}$ for each constant $\Delta \in B_{\Delta}$ : We verified this statement on a finite grid of values of $\left(\delta_{1}, \delta_{2}\right),\left|\delta_{i}\right| \leq 1$. This Lyapunov function does not depend on $\delta_{1}$ since the corresponding block of $D_{v}(z)$ is a constant. Fig. 1 shows the unit level sets of this Lyapunov function as $\delta_{2}$ varies from -1 to +1 . The level sets are skewed and rotate with $\delta_{2}$.

\section{CONCLUSION}

This technical note considered robust stability with respect to structured uncertainties. If the small gain condition is satisfied with constant scalings then the uncertain system is robustly stable with respect to norm-bounded time-varying perturbations. In this case, there is a single Lyapunov function which proves stability over all possible trajectories, i.e., the system is quadratically stable. If the small gain condition is satisfied with frequency-varying scalings then the uncertain system is robustly stable with respect to norm-bounded constant perturbations. In this technical note we constructed a PDLF which proves robust stability 


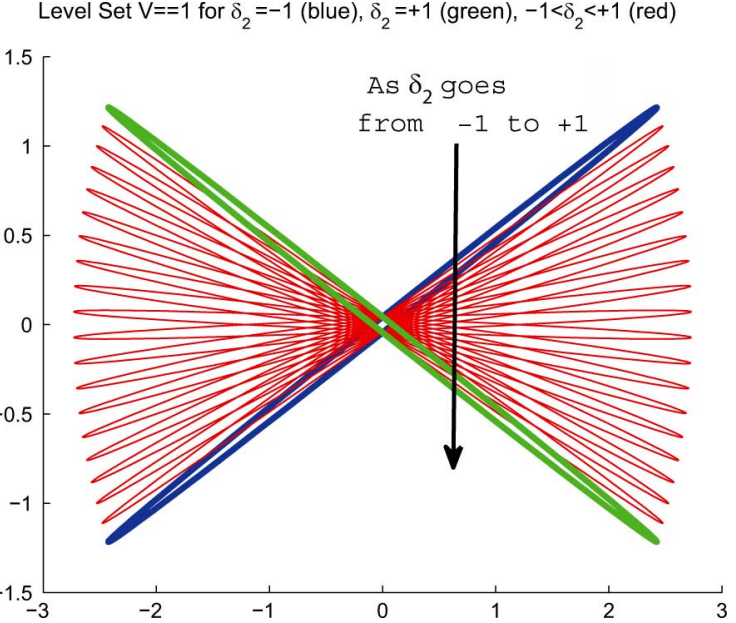

Fig. 1. Level Sets $\{x: V(x, \Delta)=1\}$ for $\delta_{2} \in[-1,1]$.

with respect to constant uncertainties. This Lyapunov function has a rational quadratic dependence on the uncertainties. It might prove fruitful to use this particular form to develop algorithms for stability and region of attraction analysis for nonlinear, uncertain systems. It would also be interesting to see if a similar explicit construction can be given for the frequency-varying quadratic separator condition. This would be interesting since it would provide a form for the PDLF which could be assumed without loss of generality when analyzing the robustness of linear systems with respect to constant uncertainties.

\section{ACKNOWLEDGMENT}

The views and conclusions contained herein are those of the authors and should not be interpreted as necessarily representing the official policies or endorsements, either expressed or implied, of the AFOSR or the U.S. Government.

\section{REFERENCES}

[1] G. Balas, R. Chiang, A. Packard, and M. Safonov, Robust Control Toolbox. Boston, MA: MathWorks, 2008.

[2] A. Packard and J. Doyle, "The complex structured singular value," $\mathrm{Au}$ tomatica, vol. 29, no. 1, pp. 71-109, 1993.

[3] J. Doyle, "Analysis of feedback systems with structured uncertainties," Proc. Inst. Elect. Eng., vol. 129, no. 6, pt. D, pp. 242-250, 1982.

[4] M. Safonov, "Stability margins of diagonally perturbed multivariable feedback systems," Proc. Inst. Elect. Eng., vol. 129, no. 6, pt. D, pp. 251-256, 1982.

[5] R. Braatz, P. Young, J. Doyle, and M. Morari, "Computational complexity of $\mu$ calculation," IEEE Trans. Autom. Control, vol. 39, no. 5, pp. 1000-1002, May 1994.

[6] J. Demmel, "The componentwise distance to the nearest singular matrix," SIAM J. Matrix Anal. Appl., vol. 13, no. 1, pp. 10-19, 1992.

[7] C. Desoer and M. Vidyasagar, Feedback Systems: Input-Output Properties. Philadelphia, PA: SIAM, 1975.

[8] G. Zames, "On the input-output stability of time-varying nonlinear feedback systems. Part I: Conditions using concepts of loop gain, conicity, and positivity," IEEE Trans. Autom. Control, vol. AC-11, no. 2, pp. 228-238, Apr. 1966.

[9] J. Shamma, "Robust stability with time varying structured uncertainty," IEEE Trans. Autom. Control, vol. 39, no. 4, pp. 714-724, Apr. 1994.

[10] B. R. Barmish, "Necessary and sufficient conditions for quadratic stabilizability of an uncertain system," J. Optim. Theory Appl., vol. 46, no. 4, pp. 399-408, 1985.

[11] S. Boyd and Q. Yang, "Structured and simultaneous Lyapunov functions for system sytability problems," Int. J. Control, vol. 49, no. 6, pp. 243-262, 1989.
[12] A. Packard and J. Doyle, "Quadratic stability with real and complex perturbations," IEEE Trans. Autom. Control, vol. 35, no. 2, pp. 198-201, Feb. 1990.

[13] P. Khargonekar, "Robust stabilization of uncertain linear systems Quadratic stabilizability and $H_{\infty}$ control theory," IEEE Trans. Autom. Control, vol. 35, no. 3, pp. 356-361, Mar. 1990.

[14] S. Boyd, L. E. Ghaoui, E. Feron, and V. Balakrishnan, Linear Matrix Inequalities in System and Control Theory. Philadelphia, PA: SIAM, 1994.

[15] M. Corless, Variable Structure and Lyapunov Control, Ser. Lecture Notes in Control and Information Sciences, Ch. Robust Stability Analysis and Controller Design With Quadratic Lyapunov Functions. New York: Springer, 1994, pp. 181-203.

[16] K. Poolla and A. Tikku, "Robust performance against time varying structured perturbations," IEEE Trans. Autom. Control, vol. 40, no. 9, pp. 1589-1602, Sep. 1995.

[17] W. Haddad and D. Bernstein, "Parameter-dependent Lyapunov functions and the Popov criterion in robust analysis and synthesis," IEEE Trans. Autom. Control, vol. 40, no. 3, pp. 536-543, Mar. 1995.

[18] S. Dasgupta, G. Chockalingam, B. Anderson, and M. Fu, "Lyapunov functions for uncertain systems with applications to the stability of time varying systems," IEEE Trans. Circuits Syst., vol. 41, no. 2, pt. I, pp. 93-106, Feb. 1994.

[19] W. Haddad and D. Bernstein, "Parameter-dependent Lyapunov functions and the discrete-time Popov criterion for robust analysis," Automatica, vol. 30, no. 6, pp. 1015-1021, 1994.

[20] E. Feron, P. Apkarian, and P. Gahinet, "S-procedure for the analysis of control systems with parametric uncertainties via parameter-dependent Lyapunov functions," in Proc. ACC, 1995, pp. 968-972.

[21] E. Feron, P. Apkarian, and P. Gahinet, "Analysis and synthesis of robust control systems via parameter-dependent Lyapunov functions," IEEE Trans. Autom. Control, vol. 41, no. 7, pp. 1041-1046, Jul. 1996.

[22] P. Gahinet, P. Apkarian, and M. Chilali, "Affine parameter-dependent Lyapunov functions and real parametric uncertainty," IEEE Trans. Autom. Control, vol. 41, no. 3, pp. 436-442, Mar. 1996.

[23] M. de Oliveira, J. Bernussou, and J. Geromel, "A new discrete-time robust stability condition,” Syst. Control Lett., vol. 37, no. 4, pp. 261-265, 1999.

[24] J. Daafouz and J. Bernussou, "Parameter dependent Lyapunov functions for discrete time systems with time varying parametric uncertainties," Syst. Control Lett., vol. 43, no. 5, pp. 355-359, 2001.

[25] M. de Oliveira and J. Geromel, "A class of robust stability conditions where linear parameter dependence of the Lyapunov function is a necessary condition for arbitrary parameter dependence," Syst. Control Lett., vol. 54, no. 11, pp. 1131-1134, 2005.

[26] M. Fu and S. Dasgupta, Advances in Linear Matrix Inequality Methods in Control. Ch. Parametric Lyapunov Functions for Uncertain Systems: The Multiplier Approach. Philadelphia, PA: SIAM, 2000, pp. 95-108.

[27] A. Trofino and C. de Souza, "Bi-quadratic stability of uncertain linear systems," in Proc. IEEE CDC, 1999, pp. 5016-5021.

[28] P. Bliman, "Nonconservative LMI approach to robust stability for systems with uncertain parameters," in Proc. IEEE CDC, 2002, pp. 305-310.

[29] G. Chesi, A. Garulli, A. Tesi, and A. Vicino, "Robust stability of polytopic systems via polynomially parameter-dependent Lyapunov functions," in Proc. IEEE CDC, 2003, pp. 4670-4675.

[30] G. Chesi, A. Garulli, A. Tesi, and A. Vicino, "Polynomially parameter-dependent Lyapunov functions for robust stability of polytopic systems: An LMI approach," IEEE Trans. Autom. Control, vol. 50, no. 3, pp. 365-370, Mar. 2005

[31] M. de Oliveira and P. Peres, "LMI conditions for robust stability analysis based on polynomially parameter-dependent Lyapunov functions," Syst. Control Lett., vol. 55, no. 1, pp. 52-61, 2006.

[32] M. Dettori and C. Scherer, "Robust stability analysis for parameter dependent systems using full-block S-procedure," in Proc. IEEE CDC, 1998, pp. $2798-2799$.

[33] M. Dettori and C. Scherer, "New robust stability and performance conditions based on parameter dependent multipliers," in Proc. IEEE CDC, 2000, pp. 4187-4192.

[34] D. Henrion, D. Arzelier, D. Peaucelle, and J. Lasserre, "On parameterdependent Lyapunov functions for robust stability of linear systems," in Proc. IEEE CDC, 2004, pp. 887-892.

[35] X. Zhang, P. Tsiotras, and T. Iwasaki, "Parameter-dependent Lyapunov functions for exact stability analysis of single-parameter dependent LTI systems," in Proc. IEEE CDC, 2003, pp. 5168-5173.

[36] A. Rantzer, "On the kalman-yakubovich-popov lemma," Syst. Control Lett., vol. 28, no. 1, pp. 7-10, 1996.

[37] M. Fan, A. Tits, and J. Doyle, "Robustness in the presence of mixed parametric uncertainty and unmodeled dynamics," IEEE Trans. Autom. Control, vol. 36, no. 1, pp. 25-38, Jan. 1991. 
[38] G. Meinsma, Y. Shrivastava, and M. Fu, "A dual formulation of mixed $\mu$ and the losslessness of (D,G) scaling," IEEE Trans. Autom. Control, vol. 42, no. 7, pp. 1032-1036, Jul. 1997.

[39] T. Iwasaki, "Robust stability analysis with quadratic separator: Parametric time-varying uncertainty case," in Proc. IEEE CDC, 1997, pp. $4880-4885$

[40] T. Iwasaki, "LPV system analysis with quadratic separator," in Proc. IEEE CDC, 1998, pp. 3021-3026.

[41] T. Iwasaki and S. Hara, "Well-posedness of feedback systems: insights into exact robustness analysis and approximate computations," IEEE Trans. Autom. Control, vol. 43, no. 5, pp. 619-630, May 1998.

[42] T. Iwasaki and G. Shibata, "LPV system analysis via quadratic separator for uncertain implicit systems," IEEE Trans. Autom. Control, vol. 46, no. 8, pp. 1195-1208, Aug. 2001.

[43] T. Kailath, A. Sayed, and B. Hasibi, Linear Estimation. Englewood Cliffs, NJ: Prentice-Hall, 2000.

[44] M. Vidyasagar, Nonlinear Systems Analysis. Philadelphia, PA: SIAM, 2002.

[45] J. Sturm, "Using SeDuMi 1.02, a MATLAB toolbox for optimization over symmetric cones," Optim. Meth. Softw., pp. 625-653, 1999.

[46] A. Trofini, "Robust stability and domain of attraction of uncertain nonlinear systems," in Proc. ACC, 2000, pp. 3707-3711.

[47] G. Chesi, "Estimating the domain of attraction for uncertain polynomial systems," Automatica, vol. 40, pp. 1981-1986, 2004.

[48] W. Tan, "Nonlinear Control Analysis and Synthesis Using Sum-ofSquares Programming," Ph.D. dissertation, Univ. California, Berkeley, 2006.

[49] D. Zhao, J. Wang, E. Poh, and F. Liao, "Stability analysis and controller synthesis for parameter-dependent polynomial nonlinear systems," in Proc. ACC, 2007, pp. 6015-6020.

[50] P. Bliman, "An existence result for polynomial solutions of parameterdependent LMIs," Syst. Control Lett., vol. 51, no. 3-4, pp. 165-169, 2004.

\section{Conditions for Synchronizability in Arrays of Coupled Linear Systems}

\author{
S. Emre Tuna
}

\begin{abstract}
Synchronization in arrays of coupled continuous-time linear systems is studied. Sufficiency of certain conditions for the existence of a synchronizing feedback law are analyzed. It is shown that, for neutrally stable systems that are detectable from their outputs, a linear feedback law exists under which any number of coupled systems synchronize provided that the (directed, weighted) graph describing the interconnection is fixed and connected. An algorithm generating one such feedback law as well as the trajectory that the solutions converge to are presented. It is also shown that, for critically unstable systems, detectability is not sufficient, whereas full-state coupling is, for the existence of a linear feedback law that is synchronizing for all coupling configurations described by a connected graph.
\end{abstract}

Index Terms-Linear systems, multi-agent system, synchronization stability, synchronizing feedback law.

\section{INTRODUCTION}

An intensively-studied problem in control theory is to find general conditions for synchronization of coupled systems, where by synchronization we mean the convergence of the solutions of systems to a common trajectory [1]-[4]. When this trajectory is a stationary point,

Manuscript received June 18, 2008; revised April 03, 2009. First published September 18, 2009; current version published October 07, 2009. Recommended by Associate Editor C. J. Tomlin.

The author is with the Department of Electrical and Electronics Engineering, Middle East Technical University, Ankara, Turkey (e-mail: tuna@eee.metu.edu. tr).

Digital Object Identifier 10.1109/TAC.2009.2029296 terms consensus or agreement can replace synchronization. See, for instance, [5]-[10]. In this note we consider linear systems with identical dynamics, interconnected over a fixed graph. We establish some new sufficient conditions for synchronization aiming to make the general picture on coupled linear systems closer to complete.

\section{A. Preliminaries}

Let $\mathbb{N}$ denote the set of nonnegative integers and $\mathbb{R} \geq 0$ set of nonnegative real numbers. Let $|\cdot|$ denote (induced) 2-norm. For $\lambda \in \mathbb{C}$ let $\operatorname{Re}(\lambda)$ denote the real part of $\lambda$. Identity matrix in $\mathbb{R}^{n \times n}$ is denoted by $I_{n}$. Matrix $A \in \mathbb{R}^{n \times n}$ is neutrally stable if it has no eigenvalue with positive real part and the Jordan block corresponding to any eigenvalue on the imaginary axis is of size one. Let $1 \in \mathbb{R}^{p}$ denote the vector with all entries equal to one. Kronecker product of two matrices $A$ and $B$ is denoted by $A \otimes B$.

A (directed) graph is a pair $(\mathcal{N}, \mathcal{E})$ where $\mathcal{N}$ is a nonempty finite set (of nodes) and $\mathcal{E}$ is a finite collection of ordered pairs (edges) $\left(n_{i}, n_{j}\right)$ with $n_{i}, n_{j} \in \mathcal{N}$. A path from $n_{1}$ to $n_{\ell}$ is a sequence of nodes $\left(n_{1}, n_{2}, \ldots, n_{\ell}\right)$ such that $\left(n_{i}, n_{i+1}\right)$ is an edge for $i \in\{1,2, \ldots, \ell-$ $1\}$. A graph is connected if it has a node to which there exists a path from every other node. ${ }^{1}$

A matrix $\Gamma:=\left[\gamma_{i j}\right] \in \mathbb{R}^{p \times p}$ describes (is) an interconnection if $\gamma_{i j} \geq 0$ for $i \neq j$ and $\gamma_{i i}=-\sum_{j \neq i} \gamma_{i j}$. It immediately follows that $\lambda=0$ is an eigenvalue with eigenvector $\mathbf{1}$ (i.e., $\Gamma \mathbf{1}=0$.) The graph of $\Gamma$ is the pair $(\mathcal{N}, \mathcal{E})$ where $\mathcal{N}=\left\{n_{1}, n_{2}, \ldots, n_{p}\right\}$ and $\left(n_{i}, n_{j}\right) \in \mathcal{E}$ iff $\gamma_{i j}>0$. Interconnection $\Gamma$ is said to be connected if its graph is connected.

Consider a connected interconnection $\Gamma$. The eigenvalue $\lambda=0$ is then distinct and all the other eigenvalues of $\Gamma$ have real parts strictly negative. When we write $\operatorname{Re}\left(\lambda_{2}(\Gamma)\right)$ we mean the real part of the nonzero eigenvalue of $\Gamma$ closest to the imaginary axis. Let $r \in \mathbb{R}^{p}$ be the left eigenvector of the eigenvalue $\lambda=0$ (i.e., $r^{T} \Gamma=0$ ). Vector $r$ is unique up to scaling since eigenvalue $\lambda=0$ is distinct. Let $r$ be scaled so that $r^{T} \mathbf{1}=1$. Then $r$ satisfies $\lim _{t \rightarrow \infty} e^{\Gamma t}=\mathbf{1} r^{T}$.

Given maps $\xi_{i}: \mathbb{R}_{\geq 0} \rightarrow \mathbb{R}^{n}$ for $i=1,2, \ldots, p$ and a map $\bar{\xi}$ : $\mathbb{R}_{\geq 0} \rightarrow \mathbb{R}^{n}$, the elements of the set $\left\{\xi_{i}(\cdot): i=1,2, \ldots, p\right\}$ are said to synchronize to $\bar{\xi}(\cdot)$ if $\left|\xi_{i}(t)-\bar{\xi}(t)\right| \rightarrow 0$ as $t \rightarrow \infty$ for all $i$. The elements of the set $\left\{\xi_{i}(\cdot): i=1,2, \ldots, p\right\}$ are said to synchronize if they synchronize to some $\bar{\xi}(\cdot)$.

Let $\mathcal{P}$ denote the set of all pairs $(C, A)$ where matrix $C$ and square matrix $A$, both real, have the same number of columns. We define the following subsets of $\mathcal{P}$.

- $\mathcal{A}_{\mathrm{H}}$ : set of all pairs $(C, A)$ with $A$ Hurwitz.

- $\mathcal{A}_{\mathrm{N}}$ : set of all pairs $(C, A)$ with $A$ neutrally stable.

- $\mathcal{A}_{\mathrm{J}}$ : set of all pairs $(C, A)$ with $A$ having no eigenvalue with positive real part.

- $\mathcal{O}_{\mathrm{F}}$ : set of all pairs $(C, A)$ with $C$ full column rank.

- $\mathcal{O}_{\mathrm{P}}$ : set of all detectable pairs.

Few remarks are in order regarding the above definitions. Note that $\mathcal{A}_{\mathrm{H}} \subset \mathcal{A}_{\mathrm{N}} \subset \mathcal{A}_{\mathrm{J}}$ and $\mathcal{O}_{\mathrm{F}} \subset \mathcal{O}_{\mathrm{P}}$. Set $\mathcal{A}_{\mathrm{J}}$ allows $A$ matrices with Jordan blocks of arbitrary size with eigenvalues on the imaginary axis, e.g., a double integrator. (Hence the subscript J.) Therefore there are pairs $(C, A)$ in $\mathcal{A}_{\mathrm{J}}$ with critically unstable $A$ matrices. For a pair $(C, A) \in \mathcal{O}_{\mathrm{F}}$, if $C$ is an output matrix of some system of order $n$ then there exists matrix $L$ such that $L C=I_{n}$, i.e., full state information is instantly available at the output. (Hence the subscript $F$.) For an arbitrary $(C, A) \in \mathcal{O}_{\mathrm{P}}$, however, the state information is only partially available at the output. (Hence the subscript P.) We also need the following notation.

- $\mathcal{G}_{\geq \delta}$ : set of all connected interconnections with $\left|\operatorname{Re}\left(\lambda_{2}(\Gamma)\right)\right| \geq$ $\delta>0$.

${ }^{1}$ This is another way of saying that the graph contains a spanning tree. 